PREPARED FOR THE U.S. DEPARTMENT OF ENERGY, UNDER CONTRACT DE-AC02-76CH03073

PPPL-3624

PPPL-3624

UC-70

Trapped-particle Instability Leading to Bursting in Stimulated Raman Scattering Simulations

by

S. Brunner and E. Valeo

November 2001

NM|

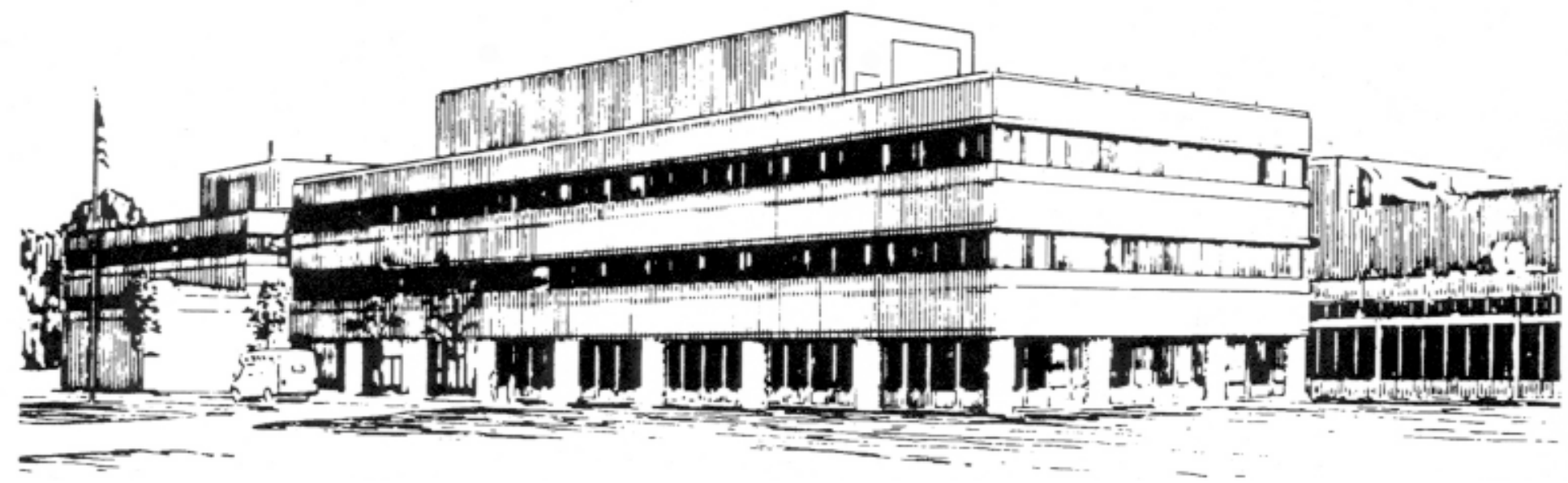

PRINCETON PLASMA PHYSICS LABORATORY PRINCETON UNIVERSITY, PRINCETON, NEW JERSEY 


\section{PPPL Reports Disclaimer}

This report was prepared as an account of work sponsored by an agency of the United States Government. Neither the United States Government nor any agency thereof, nor any of their employees, makes any warranty, express or implied, or assumes any legal liability or responsibility for the accuracy, completeness, or usefulness of any information, apparatus, product, or process disclosed, or represents that its use would not infringe privately owned rights. Reference herein to any specific commercial product, process, or service by trade name, trademark, manufacturer, or otherwise, does not necessarily constitute or imply its endorsement, recommendation, or favoring by the United States Government or any agency thereof. The views and opinions of authors expressed herein do not necessarily state or reflect those of the United States Government or any agency thereof.

\section{Availability}

This report is posted on the U.S. Department of Energy's Princeton Plasma Physics Laboratory Publications and Reports web site in Fiscal Year 2002. The home page for PPPL Reports and Publications is: http://www.pppl.gov/pub_report/

DOE and DOE Contractors can obtain copies of this report from:

U.S. Department of Energy

Office of Scientific and Technical Information

DOE Technical Information Services (DTIS)

P.O. Box 62

Oak Ridge, TN 37831

Telephone: (865) 576-8401

Fax: (865) 576-5728

Email: reports@adonis.osti.gov

This report is available to the general public from:

National Technical Information Service

U.S. Department of Commerce

5285 Port Royal Road

Springfield, VA 22161

Telephone: 1-800-553-6847 or

(703) 605-6000

Fax: (703) 321-8547

Internet: http://www.ntis.gov/ordering.htm 


\title{
Trapped-Particle Instability Leading to Bursting in Stimulated Raman Scattering Simulations
}

\author{
S. Brunner* and E. Valeo \\ Princeton Plasma Physics Laboratory, P.O. Box 451, Princeton, New Jersey 08543-0451
}

(October 26, 2001)

\begin{abstract}
Non-linear, kinetic simulations of Stimulated Raman Scattering (SRS), for laser-fusion relevant conditions, present a bursting behavior. Different explanations for this regime had been given in previous studies: Saturation of SRS by increased non-linear Landau damping [K. Estabrook et al., Phys. Fluids B 1, 1282 (1989)], and detuning due to the non-linear frequency shift of the plasma wave [H. X. Vu et al., Phys. Rev. Lett. 86, 4306 (2001)]. Another mechanism, also assigning a key role to the trapped electrons is proposed here: The break-up of the plasma wave through the trapped-particle instability. 52.38.Bv, 52.35.Mw, 52.65.Ff
\end{abstract}

Typeset using REVTEX

*E-mail: sbrunner@pppl.gov 
A more thorough understanding of the dynamics of parametric instabilities is essential, in order to improve the existing tools used for designing laser-fusion systems [1]. Stimulated Brillouin scattering (SBS) and stimulated Raman scattering (SRS) are the basic two such instabilities affecting the laser beam, respectively involving the scattering off of ion-acoustic waves (IAWs) and electron plasma waves (EPWs). In the following, we shall essentially address issues involving the non-linear evolution of SRS.

The bursting behavior of SRS appears as a common characteristic of different non-linear, kinetic simulations $[2,3]$ : Instead of reaching a steady-state, with constant intensity of the reflected light, SRS presents an ongoing dynamical evolution, building up and breaking down in cycles. In these previous studies, different mechanisms have been suggested for this intermittence. The non-linear wave-particle interaction has, however, always been identified as playing a key role: Estabrook, Kruer, and Haines [2] tentatively propose the saturation of SRS by increased non-linear Landau damping, due to hot electrons generated through the trapping process, while $\mathrm{Vu}$, DuBois, and Bezzerides [3] recently interpreted their results by proposing that trapped particles lead to a significant non-linear shift in the EPW frequency, which then detunes the parametric instability.

On the basis of new simulations, a third possible mechanism for explaining the pulsating of SRS is presented in this paper, also assigning an essential role to trapped electrons: The break-up of the EPW through a secondary instability, the so-called trapped-particle instability (TPI), first described by Kruer, Dawson, and Sudan [4].

The results are based on a spatially one-dimensional model with open boundaries, which represents the laser-plasma interaction along the beam. By solving the Vlasov equation for electrons in the phase space $\left(x, v_{x}\right)$, where $x$ and $v_{x}$ are, respectively, the position and velocity along the laser beam, the simulations are fully kinetic in the longitudinal direction. In this way, the essential kinetic effect, i.e. the non-linear trapping in the electrostatic plasma wave, is taken account of. The Vlasov equation is solved numerically by applying the Eulerian scheme [5], which evolves the particle distribution on a fixed Cartesian mesh in phase space, and thus avoids the numerical noise affecting particle in cell (PIC)-type 
approaches, as used in Refs. [2] and [3]. As a result, very "clean" simulations are achieved, which are straightforward to diagnose. For the transverse motion, and so as to lighten the computations, the plasma is assumed cold, so that a fluid-like description can be considered. As the study is limited to SRS, ions are assumed immobile, providing a uniform, neutralizing background. Hence, the simulation model is quite similar to the one described in Ref. [6]. Two antennas are implemented in the system: The first, at the left edge, for launching the laser light, the second, at the right edge, for emitting the seed for the scattered light. This second antenna thus replaces the actual physical seed, i.e. the electromagnetic thermal noise fluctuations, not contained as such in our model.

The physical parameters are inspired by the recent single laser hot spot experiments [7], carried out at the Los Alamos National Laboratory (LANL). The initial electron temperature is thus chosen $T=550 \mathrm{eV}$, and the density is assumed to be $5 \%$ of the critical density at the considered laser wavelength in vacuum $\lambda_{0}^{\mathrm{vac}}=0.5 \mu$. By solving the matching condition equations for backward SRS [10], one obtains $\left(\omega_{0} / \omega_{p}=4.472, k_{0} \lambda_{D}=0.143\right)$, $\left(\omega_{s} / \omega_{p}=3.383, k_{s} \lambda_{D}=-0.106\right)$, and $\left(\omega_{e} / \omega_{p}=1.089, k_{e} \lambda_{D}=0.249\right)$ for the frequencies and wavenumbers of the incident light, scattered light, and EPW respectively. Frequencies are normalized with respect to the plasma frequency $\omega_{p}$, and lengths with respect to the Debye length $\lambda_{D}$. Simulations were carried out for a set of incoming laser intensities in the range $I_{0}=2.5 \cdot 10^{14}-1.6 \cdot 10^{16} \mathrm{Wcm}^{-2}$. The intensity of the scattering seed is arbitrarily chosen to be $I_{s}^{\text {seed }}=10^{-4} I_{0}$. Furthermore, the effective interaction length of the simulation system, amounting to the distance between the two antennas, is $L_{\mathrm{eff}}=1730 \lambda_{D} \simeq 40 \lambda_{0}$, which turns out to be only a fraction of the experimental hot spot length.

The initial electron distribution is assumed Maxwellian. Hence, non-classical transport effects, which may lead to significant deviations from such a distribution under the conditions considered here [8], and, in turn, to quantitative changes in the thresholds and gains of parametric instabilities [9], are neglected for simplicity, as they do not qualitatively alter the bursting behavior of SRS.

The particular case of incoming laser intensity $I_{0}=4 \cdot 10^{15} \mathrm{Wcm}^{-2}$ will now be considered 
in detail. Let us emphasize, that the following observations and interpretations have also shown to be valid for the whole set of simulations carried out over the range $I_{0}=2.5 \cdot 10^{14}-$ $1.6 \cdot 10^{16} \mathrm{Wcm}^{-2}$.

The reflectivity as a function of time, is shown in Fig. 1 for the whole simulation, which lasts $1400 \omega_{p}^{-1}$. Clearly, SRS presents a bursting behavior: The intensity of the reflected light successively increases, peaks, and then drops sharply to relatively low levels. This cycle repeats itself with a certain regularity, the recovery period in this case being approximately $400-500 \omega_{p}^{-1}$. The evolution of SRS thus appears to be an ongoing, dynamic process.

The average reflectivity over the the whole simulation is $\langle R\rangle=3.5 \%$, while peak values are $2.5-5$ times larger. Let us point out, that the absolute values of reflectivity are not necessarily realistic, as the simulation system is shorter than the actual hot spot, and the seed for the scattered light has been chosen arbitrarily.

A view, of what is occuring within the interaction region during the SRS cycles, is given in Fig. 2, where the electrostatic field $E_{\mathrm{es}, \mathrm{x}}$ of the EPW is plotted as a function of position and time. Figure 2.a is a low resolution plot, showing the amplitude of the field over the full length $L$ of the simulation, and for the time interval $200<t \omega_{p}<1000$, which includes the first two bursts. Note the positions in Fig. 2.a of antennas 1 and 2, emitting respectively the laser light and seed for the scattered light.

Considering the first SRS cycle, the field $E_{\mathrm{es}, \mathrm{x}}$ progressively builds up until time $t \omega_{p} \simeq$ 350, in conjunction with increasing reflectivity (Fig. 1). Up till the end of this growth stage, the EPW remains a coherent field. Only a slight, periodic modulation of the field's envelope is to be noted. In particular, as shown clearly in Fig. 3.a for $t \omega_{p}=350$, the essentially monochromatic wave enables strong trapping of the resonant electrons.

After time $t \omega_{p} \simeq 350$, the EPW starts to break up in the large amplitude region $300 \lesssim$ $x \lambda_{D} \lesssim 600$, later expanding itself to the entire field, which disintegrates into individual wavepackets, that drift off at the group velocity $v_{g, e}=3 v_{\mathrm{th}}^{2}\left(k_{e} / \omega_{e}\right)=0.69 v_{\mathrm{th}}$. At this point, the system has reached a turbulent state, reflected by the chaotic motion of previously trapped particles (see Fig. 3.b). Taking account of the time delay required for the scattered light 
to exit the system, this first break-up indeed occurs simultaneously with the first drop of reflectivity in Fig. 1. Following its disintegration, the remains of the EPW have partly damped out and convected out of the interaction zone after a time interval of $400-500 \omega_{p}^{-1}$. At this point, SRS is able to recover to some extent, leading to the second burst in reflectivity. Recovery is only partial, as the initial state for this second SRS cycle is not a fully quiescent plasma. This translates itself by the subsequent peaks in Fig. 1 being lower than the first.

A key observation was made, when repeating the same simulation, but artificially turning off the self-consistent ponderomotive drive at time $t \omega_{p}=320$, and noticing, that the EPW evolved in essentially the same way. One can therefore conclude, that the break-up is the result of an instability affecting the EPW itself, independent of the three-wave interaction of the parametric instability.

A close-up view of the break-up is given by Fig. 2.b, corresponding to the dash-bordered region in Fig. 2.a. The diagonal lines are the constant phase characteristics, traveling at the phase velocity $v_{\phi, e}$. It clearly appears from this figure, that the modulation of the initially coherent field seems to provide the seed to the instability.

The origin of the modulation can be identified, by considering the electron velocity distribution function, averaged over one wavelength $\lambda_{e}=2 \pi / k_{e}$, at two different points in space and time, one corresponding to a maximum, the other to a minimum in the modulation. Two such points have been chosen in Fig. 2.b, and the corresponding averaged distributions are given in Fig. 4. Obviously, the distribution inverts itself at the resonant phase velocity $v_{\phi, e}$, when going from a maximum to a minimum of the modulation, reflecting different bounce phases of the trapped electrons.

The average bounce-period $\tau_{b}$ can thus be estimated, by measuring the time along a given phase characteristic, separating two extremas in the field envelope. Points 1 and 2 in Fig. 2.b happen to have been chosen along the same characteristic, and are therefore separated by approximately $\tau_{b} / 2$, so that $\tau_{b} \omega_{p} \simeq 20$. The wavelength $\lambda_{\text {mod }}$ of the spatial modulation is directly related to the bounce-period, by the relation $\lambda_{\text {mod }} \simeq v_{\phi, e} \tau_{b} \simeq 90 \lambda_{D}$.

The spatial modulation naturally translates itself in Fourier space by the presence of 
sidebands $k_{e} \pm \Delta k_{\text {mod }}$ to the main plasma mode $k_{e}$, where $\Delta k_{\text {mod }}=2 \pi / \lambda_{\text {mod }}$. The time evolution of these sidebands, appearing as peaks in the $k$-spectrum of the wave, are plotted in Fig. 5.a, showing exponential growth in the interval $350 \lesssim t \omega_{p} \lesssim 400$, characteristic of the linear stage of an instability. From these curves, the growth rate of sidebands $k_{e} \pm \Delta k_{\text {mod }}$ are estimated to be $\gamma=6.1 \cdot 10^{-2} \omega_{p}$ and $\gamma=3.6 \cdot 10^{-2} \omega_{p}$ respectively.

The observations, made by studying our simulation results of SRS bursting, may thus be summarized as follows: (1) The turn-off of SRS reflectivity is the result of a secondary instability, affecting the EPW itself. (2) This instability involves sidebands of the initial large amplitude EPW. (3) The dynamics of trapped particles seem to play an essential role in the instability process. Considering these properties, the mechanism of the TPI, appears as a logical explanation for the observed event. Indeed, given a large amplitude EPW, the TPI affects sidebands driven by the electrons trapped in the troughs of the main mode. A recent review of this sideband instability is given in reference [11].

In their reduced model for the TPI, Kruer, Dawson, and Sudan [4] represent the electrons trapped within one potential well, by a single, harmonically bound macro-particle. Implemented in this simple way, trapping is the only kinetic effect included in the macroparticle model, which in all other respects is fluid-like (e.g. Landau damping of the EPW is neglected). In this framework, the dispersion relation for the TPI (Eq. (7) Ref. [4]) has three independent parameters: The wavenumber $k_{e}$ of the principal plasma wave, the bounce-frequency $\omega_{b}$, and the fraction $f_{t}$ of trapped particles.

To compare the solution of this dispersion relation, to the growth rates of the sidebands $k_{e} \pm \Delta k_{\text {mod }}$ from our simulations, these parameters are thus given the values $k_{e} \lambda_{D}=0.249$, $\omega_{b}=2 \pi / \tau_{b}=0.31 \omega_{p}$, and $f_{t}=5.9 \%, f_{t}$ having been integrated from the distribution in Fig. 3.a. The corresponding result is shown in Fig. 5.b, and presents the typical, doublehumped shape, symmetrical around the principal wavenumber $k_{e}$. The linear evolution of the simulation sidebands $k_{e} \pm \Delta k_{\text {mod }}$ agrees well with the peaks of the dispersion relation solution, both in the values of the wavenumbers and growth rates. The agreement is astonishing, considering the relative simplicity of the macro-particle model. The fact that the simulation 
growth rates are somewhat lower than predicted by the dispersion relation, can be assigned to Landau damping. Furthermore, the fact that this damping increases with $k$, explains why the mode $k_{e}+\Delta k_{\text {mod }}$ presents an even lower value than the one of mode $k_{e}-\Delta k_{\bmod }$. The good semi-quantitative agreement, with the TPI dispersion relation, was confirmed in all other simulations performed in the range $I_{0}=2.5 \cdot 10^{14}-1.6 \cdot 10^{16} \mathrm{Wcm}^{-2}$.

Further credibility, to the essential role played by the TPI in the mechanism leading to the bursting of SRS, was given, by studying a reduced simulation system, with periodic boundaries instead of open ones, and the resonant component of the self-consistent ponderomotive force replaced by an external drive $F_{x}^{\text {ext }}=(-e) E_{x}^{\text {ext }}=(-e) E_{0} \cos \left(k_{e} x-\omega_{e} t\right)$. The parameters $E_{0}=1 \cdot 10^{-2} T / e \lambda_{D}$ and $\left(\omega_{e}=1.089 \omega_{p}, k_{e} \lambda_{D}=0.249\right)$ were chosen so as to best reproduce the conditions of the full simulation. At first, the reduced system was taken just one wavelength long, $L=2 \pi / k_{e}$, so that no sidebands, besides the harmonics of the main mode, were allowed. This setup is similar to the one considered by Cohen and Kaufman [12]. In a second run, sideband effects were included, by increasing the system to the arbitrary length $L=12 \times 2 \pi / k_{e}$. To provide a seed for the possible growth of sidebands, an initial random density perturbation, with an arbitrarily chosen, relative amplitude of $\sim 0.5 \%$, was considered for all these reduced simulations.

In the single wavelength simulation, and as a result of the the non-linear frequency shift, the phase difference $\Delta \phi$, between the principal plasma wave $E_{\mathrm{es}, \mathrm{x}}$ and the drive $E_{x}^{\mathrm{ext}}$, oscillates around $\pi$, and its amplitude thus presents a harmonic modulation in time (Fig. 6.a). This is similar to the result in Fig. 4 of Ref. [12]. In the multiple wavelength simulation, however, the harmonic modulation is rapidly interrupted, the phase lock-in with the drive is lost, and the main mode $k_{e}$ depletes itself in a way comparable to the break-down of reflectivity shown in Fig. 1. This depletion occurs in conjunction with the growth of sidebands, which, after comparison with the macro-particle model, can again be related to the TPI (Fig. 6.b). The parameter estimates $\omega_{b}=0.21 \omega_{p}$ and $f_{t}=1.6 \%$, for solving the TPI dispersion relation, turn out to be nonetheless slightly different from the full simulation case. 
Even though the physical parameters for the simulations presented here were inspired by the LANL single laser hot spot experiment, the TPI may well affect the non-linear evolution of SRS over an even wider range of conditions. But it should be emphasized at the same time, that, although dominant in our computations, the TPI is not claimed to be the only possible process leading to the SRS pulsations. Indeed, as partly observed in our reduced simulations, other effects, such as detuning resulting from the non-linear frequency shift of the plasma wave [3], may also contribute, either simultaneously, or in turns.

This work was supported by the Lawrence Livermore National Laboratory under DOE Interoffice Work Order Number B344523, by the U.S. Department of Energy Contract No. DE-AC02-76-CHO-3073. 


\section{REFERENCES}

[1] J. Lindl, Phys. Plasmas 2, 3933 (1995); S. E. Bodner et al., Phys. Plasmas 5, 1901 (1998).

[2] K. Estabrook, W. L. Kruer, and M. G. Haines, Phys. Fluids B 1, 1282 (1989).

[3] H. X. Vu, D. F. DuBois, and B. Bezzerides, Phys. Rev. Lett. 86, 4306 (2001).

[4] W. L. Kruer, J. M. Dawson, and R. N. Sudan, Phys. Rev. Lett. 23, 838 (1969).

[5] C. Z. Cheng and G. Knorr, J. Comp. Phys. 22, 330 (1976).

[6] P. Bertrand et al., Phys. Fluids B 2, 1028 (1990).

[7] D. S. Montgomeryet al., Phys. Rev. Lett. 84, 678 (2000).

[8] S. Brunner and E. Valeo, "Simulations of Electron Transport in Laser Hot Spots," submitted to Phys. Plasmas.

[9] B. B. Afeyan et al., Phys. Rev. Lett. 80, 2322 (1998).

[10] W. L. Kruer, The Physics of Laser Plasma Interactions (Addison-Wesley, New York, 1988).

[11] D. A. Hartmann and C. F. Driscoll, Phys. Plasmas 8, 3457 (2001).

[12] B. I. Cohen and A. N. Kaufman, Phys. Fluids 20, 1113 (1977). 
Fig. 1 Backward SRS reflectivity as a function of time. Three burst cycles occur during the whole simulation.

Fig. 2 (a) Evolution of the electrostatic field, over the first two burst cycles of SRS. Having reached a finite amplitude, the wave breaks up in packets, drifting off at the group velocity $v_{g, e}$. (b) Close-up view of the break-up (dash-bordered region in Fig. a). Diagonal lines are formed by constant phase characteristics, traveling at the phase velocity $v_{\phi, e}$. Visible is the spatial modulation, leading to the break-up. Points 1 and 2 correspond respectively to a maximum and minimum of the modulation.

Fig. 3 Electron distribution at time (a) $t \omega_{p}=350$ and (b) $t \omega_{p}=450$ (times pointed out in Fig. 1), in a limited region of phase space $\left(x, v_{x}\right)$, corresponding to approximately 4 wavelengths of the main plasma mode. The separatrix of the trapping region is represented by dashed lines in (a).

Fig. 4 Velocity distribution, averaged in space over one wavelength $\lambda_{e}$. Going from a maximum to a minimum of the field modulation (pt. 1 \& 2 in Fig. 2, resp.), the distribution systematically inverts itself around the phase velocity $v_{\phi, e}$.

Fig. 5 (a) Time evolution of sidebands $k_{e} \pm \Delta k_{\text {mod }}$. (b) Comparing growth rates $\gamma$ of the sidebands, with the solution to the dispersion relation for the trapped-particle instability, in the framework of the macro-particle model.

Fig. 6 Reduced simulation results. (a) Amplitude of $E_{\mathrm{es}, \mathrm{x}}$ in units $T / e \lambda_{D}$ (left scale), and dephasing $\Delta \phi$ wrt. external drive $E_{x}^{\text {ext }}$ (right scale), for the $L=\lambda_{e}$ and $L=12 \lambda_{e}$ long system. (b) Comparison of sideband growth rates in $L=12 \lambda_{e}$ system, with results from macro-particle model. 
FIG. 1.

1st Author: S. Brunner

Paper: Phys. Rev. Lett.

This is the intended size for final publication.

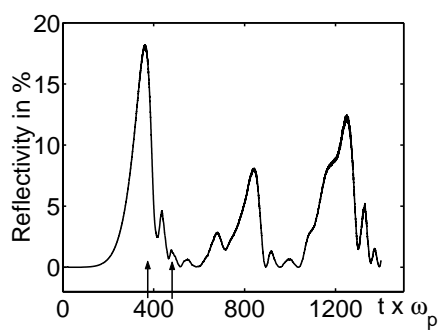


FIG. 2.

1st Author: S. Brunner

Paper: Phys. Rev. Lett.
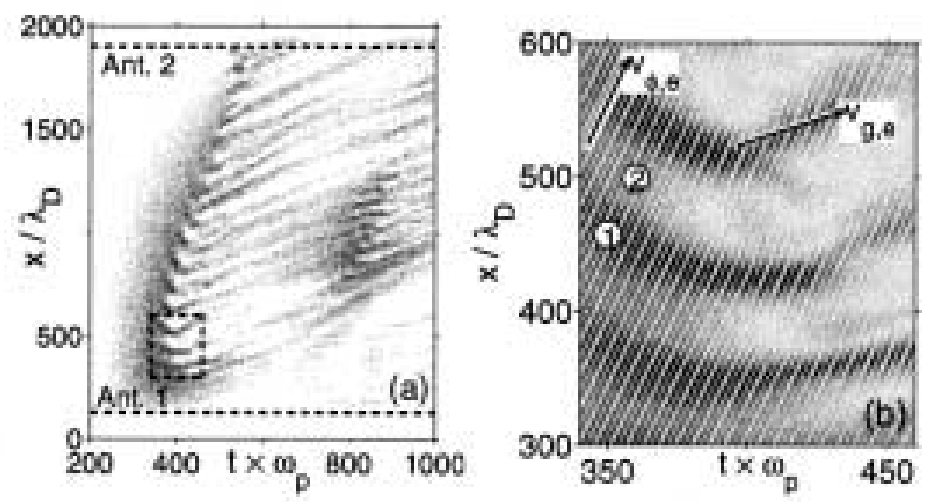
FIG. 3.

1st Author: S. Brunner

Paper: Phys. Rev. Lett.
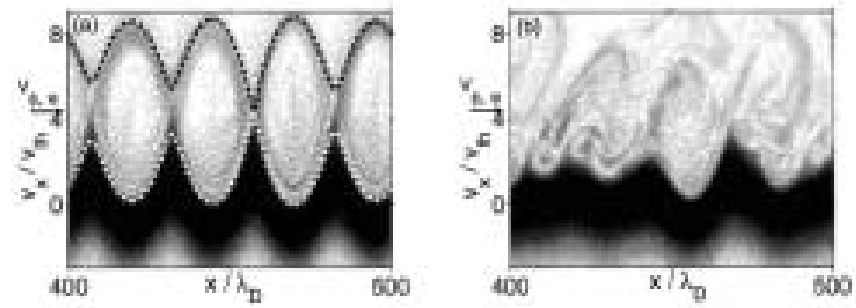
FIG. 4.

1st Author: S. Brunner

Paper: Phys. Rev. Lett.

This is the intended size for final publication.

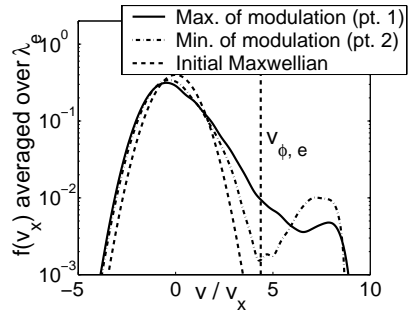


FIG. 5 .

1st Author: S. Brunner

Paper: Phys. Rev. Lett.

This is the intended size for final publication.
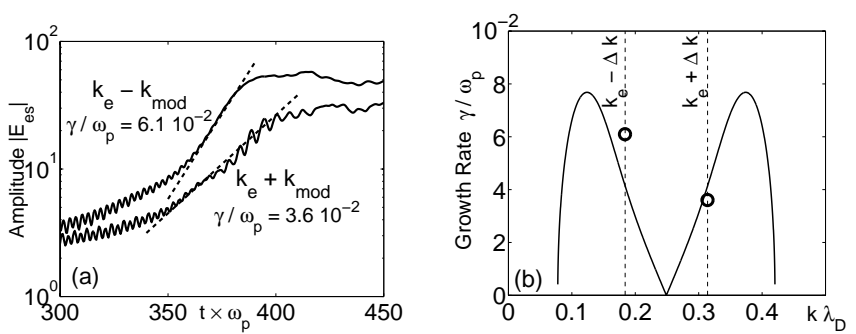
FIG. 6.

1st Author: S. Brunner

Paper: Phys. Rev. Lett.

This is the intended size for final publication.
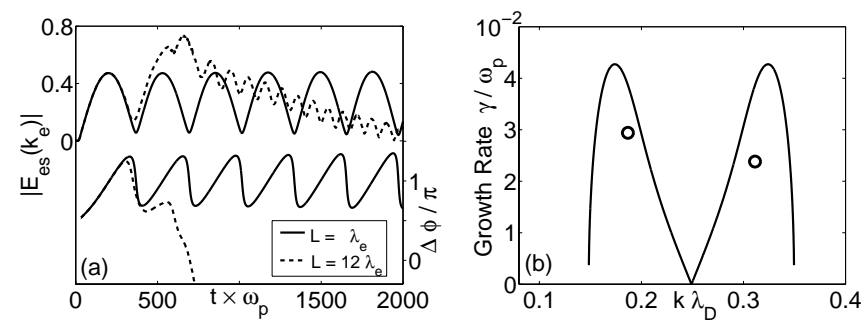


\section{External Distribution}

Plasma Research Laboratory, Australian National University, Australia

Professor I.R. J ones, Flinders University, Australia

Professor J oão Canalle, Instituto de Fisica DEQ/IF - UERJ , Brazil

Mr. Gerson O. Ludwig, Instituto Nacional de Pesquisas, Brazil

Dr. P.H. Sakanaka, Instituto Fisica, Brazil

The Librarian, Culham Laboratory, England

Library, R61, Rutherford Appleton Laboratory, England

Mrs. S.A. Hutchinson, JET Library, England

Professor M.N. Bussac, Ecole Polytechnique, France

Librarian, Max-Planck-Institut für Plasmaphysik, Germany

J olan Moldvai, Reports Library, MTA KFKI-ATKI, Hungary

Dr. P. Kaw, Institute for Plasma Research, India

Ms. P.J . Pathak, Librarian, Insitute for Plasma Research, India

Ms. Clelia De Palo, Associazione EURATOM-ENEA, I taly

Dr. G. Grosso, Instituto di Fisica del Plasma, Italy

Librarian, Naka Fusion Research Establishment, J AERI, J apan

Library, Plasma Physics Laboratory, Kyoto University, J apan

Research Information Center, National Institute for Fusion Science, J apan

Dr. O. Mitarai, Kyushu Tokai University, J apan

Library, Academia Sinica, Institute of Plasma Physics, People's Republic of China

Shih-Tung Tsai, Institute of Physics, Chinese Academy of Sciences, People's Republic of China

Dr. S. Mirnov, TRINITI, Troitsk, Russian Federation, Russia

Dr. V.S. Strelkov, Kurchatov Institute, Russian Federation, Russia

Professor Peter Lukac, Katedra Fyziky Plazmy MFF UK, Mlynska dolina F-2, Komenskeho Univerzita, SK-842 15 Bratislava, Slovakia

Dr. G.S. Lee, Korea Basic Science Institute, South Korea

Mr. Dennis Bruggink, Fusion Library, University of Wisconsin, USA

Institute for Plasma Research, University of Maryland, USA

Librarian, Fusion Energy Division, Oak Ridge National Laboratory, USA

Librarian, Institute of Fusion Studies, University of Texas, USA

Librarian, Magnetic Fusion Program, Lawrence Livermore National Laboratory, USA

Library, General Atomics, USA

Plasma Physics Group, Fusion Energy Research Program, University of California at San Diego, USA

Plasma Physics Library, Columbia University, USA

Alkesh Punjabi, Center for Fusion Research and Training, Hampton University, USA

Dr. W.M. Stacey, Fusion Research Center, Georgia Institute of Technology, USA

Dr. J ohn Willis, U.S. Department of Energy, Office of Fusion Energy Sciences, USA

Mr. Paul H. Wright, Indianapolis, Indiana, USA 
The Princeton Plasma Physics Laboratory is operated by Princeton University under contract with the U.S. Department of Energy.

\author{
Information Services \\ Princeton Plasma Physics Laboratory \\ P.O. Box 451 \\ Princeton, NJ 08543
}

Phone: 609-243-2750

Fax: 609-243-2751

e-mail: pppl_info@pppl.gov

Internet Address: http://www.pppl.gov 